\title{
A new endoscopic technique to evacuate organised blood clots from the bladder
}

\author{
M. Wijewardena ${ }^{1}$, A.M. Abeygunasekera ${ }^{2}$ \\ 1 Department of Urology, National Hospital of Sri Lanka \\ 2 Department of Urology, Colombo South Teaching Hospital, Sri Lanka
}

Key words: Bladder clots; bladder evacuation

\begin{abstract}
Massive haematuria with clot retention is a surgical emergency. The established techniques to remove such bladder clots are bladder irrigation with a three way catheter, use of a bladder syringe to suck out clots and use of a resectoscope and loops. Delayed organised clots in large amounts are difficult to be removed using these methods. We describe a new technique where a nasogastric tube is inserted via the resectoscope sheath and connected to an ordinary suction apparatus that could be used for successful removal of organised blood clots in the bladder.
\end{abstract}

\section{Introduction}

Massive haematuria resulting in clot formation inside the bladder and causing urinary retention is an occasional urological emergency encountered by surgeons in their clinical practice [1]. Such patients are in extreme distress due to pain, and evacuation of clots in the bladder becomes an urgent necessity. Bladder irrigation with a three-way urethral catheter helps to settle most cases with massive haematuria and bladder clots. However, some patients have organised clots due to prolonged bleeding as in cases of haemorrhagic cystitis after pelvic irradiation or chemotherapy. Even evacuation of the bladder under anaesthesia using standard techniques becomes a challenge in such situations. Here we describe a simple technique which helps to evacuate organised blood clots from the bladder easily and safely.

\section{Case report}

A 12-year-old boy was transferred from another institute with uncontrollable haematuria after chemotherapy with cyclophosphamide. On examination he was pale and in pain due to a distended bladder which was reaching his umbilicus. He was passing small amounts of heavily blood stained urine. The distended bladder was felt as a hard mass in the lower half of the abdomen as it was full of organised blood clots after

Correspondence: A.M. Abeygunasekera

E-mail: amabey@sltnet.lk

DOI: http://10.4038/sljs.v34i2.8265

\section{(cc) BY}

The Sri Lanka Journal of Surgery 2016; 34(2): 26-27 prolonged and heavy haematuria. The child refused to have a urethral catheter inserted as it had not helped to evacuate the bladder and had caused him much discomfort. His haemoglobin level was $7 \mathrm{~g} / \mathrm{dl}$ and was corrected with urgent blood transfusions. A diagnosis of blood clots in the bladder was made and bladder washout under anaesthesia was planned.

Cystoscopy under general anaesthesia confirmed the presumptive diagnosis of large amounts of old blood clots in the bladder. Cystoscopic vision was poor due to the large blood clots filling the entire bladder. Many attempts to evacuate the clots using the Ellik evacuator failed. Next, resecting loops were used to remove the clots but could remove only a small amount of clots [1]. As there were large amounts of hardened clots left, and prolonged anaesthesia and bladder irrigation could cause deleterious effects, we adopted a new technique to remove clots. The new technique was similar to the method used to remove blood clots and stone fragments during a percutaneous nephrolithotomy (PCNL). Through the resectoscope sheath we inserted a nasogastric tube (12 Fr) into the bladder after truncating its tip. The nozzle of the nasogastric tube was connected to a sucker handle, connected to the tube of an ordinary sucker machine used in the operating room. Suction was applied while irrigation fluid was continuously infused through the normal irrigating channel of the resectoscope sheath. The gap between resectoscope sheath and the nasogastric tube was closed with the fingers to minimise the irrigant fluid leakage. Within a minute or two we managed to evacuate all the clots and subsequent cystoscopy confirmed a bladder devoid of any clots. There were no mucosal lesions suggestive of a bladder malignancy and the most likely cause for the child's haematuria was established as drug induced haemorrhagic cystitis. An 18 Fr three way catheter was left in place to continue bladder irrigation with saline. After 24 hours the catheter was removed as urine remained clear. The patient left the hospital the next day.

\section{Discussion}

The common causes of massive and prolonged haematuria are post irradiation cystitis, drug induced haemorrhagic cystitis, bladder malignancies, blood dyscrasias and even infections in 
the presence of anticoagulants [1]. Bleeding from the upper urinary tract (e.g. after renal surgery, renal tumours, arterioureteral fistulae) and prostatic or bladder surgery also can cause massive haematuria. These patients may develop urinary retention due to bladder clots and can be in extreme discomfort. The pain due to inability to pass urine becomes the main problem as blood loss can be treated with blood transfusions. Evacuation of these bladder clots pose a problem, and bladder irrigation with a three way catheter is the procedure of choice to overcome the problem [1]. However in cases of prolonged bleeding, clots become organised and hard, and irrigation combined with aspiration of clots with a bladder syringe may fail to dislodge such clots. Evacuation of such organised clots becomes difficult even under anaesthesia using standard bladder evacuators and resectoscope loops. There are instances where open vesicotomy has been done to evacuate such clots. There is morbidity associated with such open surgery. Furthermore such open procedures are contraindicated in cases of bladder malignancy due to the possibility of implantation of tumour tissue. These patients develop haematuria and blood clots repeatedly as the underlying cause cannot be corrected on a permanent basis, hence an endoscopic procedure will be much better than an open procedure.

We have tried this new technique in several patients with success and without any unwanted side effects. However, applying extreme suction may cause inversion and damage to the bladder wall and should be avoided. We hope this new technique would be of use in the management of cases with organised blood clots in the bladder which are resistant to the standard methods of clot evacuation.

\section{References}

1. Thevarajah Aravinthan. Massive haematuria with clot retention: management options. Sri Lanka J Surg 2013; 30:18-19

2. Romao AJ, Guerreiro MG, Lopes MM. Hyperbaric oxygen therapy for refractory radiation-induced hemorrhagic cystitis. Int JUrol 2015; 22: 962-966

http://dx.doi.org/10.1111/iju.12857

\section{IMAGES IN SURGERY}

\section{P. Sethi, T. Manoj, O.V. Sudheer}

Department of Gastrointestinal Surgery, Amrita Institute of Medical Sciences, India.

\section{Case:}

A 45 year old female presented with profuse malaena and haemodynamic instability. Gastroduodenoscopy revealed clots in the third part of the duodenum with no localisation of the bleed. An MD-CT abdomen revealed minimal intraluminal contrast extravasation in the proximal jejunum with an intramural hematoma. A conventional angiogram surprisingly revealed a globular lesion in the proximal jejunum with extensive blush (Figure 1). Percutaneous transfemoral angioembolisation of the feeding vessel (first jejunal branch of SMA) was done to control the bleeding. Despite these efforts, haemodynamic instability persisted and she underwent a laparotomy which revealed a highly vascular proximal jejunal mass (Figure 2), which was excised and a jejuno-jejunal anastomosis was fashioned. Immunostaining of the excised mass was strongly positive for CD117 and DOG1.

\section{What is the diagnosis?}

2. What is the management of this disease?

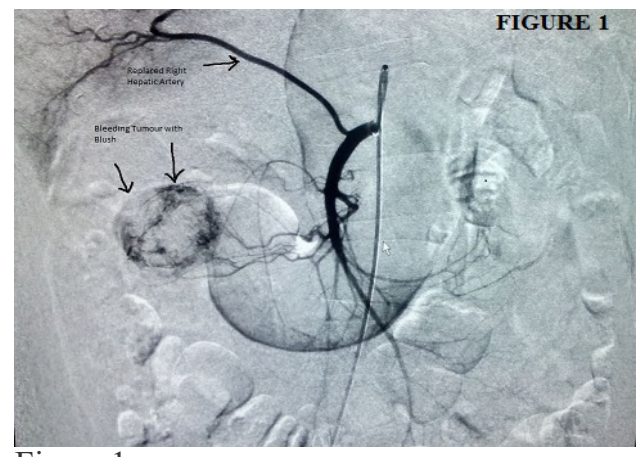

Figure 1

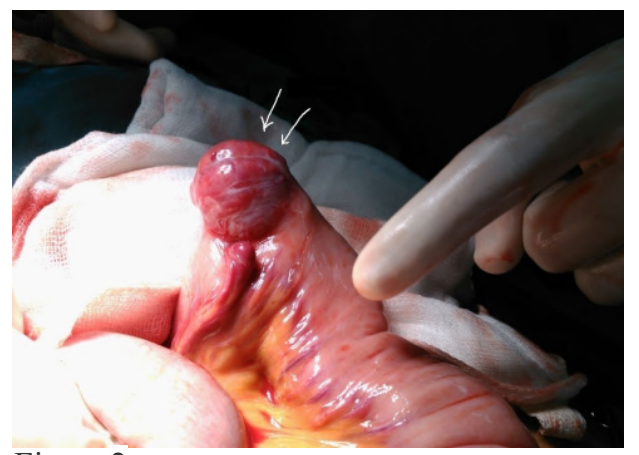

Figure 2

Correspondence: P. Sethi

E-mail: drpulkitsethi@gmail.com

DOI: http://10.4038/sljs.v34i2.8266 